\title{
The simple loop conjecture for 3-manifolds modeled on Sol
}

\author{
DREW ZEMKE
}

\begin{abstract}
The simple loop conjecture for 3-manifolds states that every 2-sided immersion of a closed surface into a 3-manifold is either injective on fundamental groups or admits a compression. This can be viewed as a generalization of the loop theorem to immersed surfaces. We prove the conjecture in the case that the target 3-manifold admits a geometric structure modeled on Sol.
\end{abstract}

57M35; 57M50

\section{Introduction}

The simple loop conjecture for 3-manifolds is as follows.

Conjecture [7, Problem 3.96] Let $\Sigma$ be a closed surface and let $M$ be a closed 3-manifold. If $F: \Sigma \rightarrow M$ is a 2-sided immersion for which the induced map $F_{*}: \pi_{1} \Sigma \rightarrow \pi_{1} M$ is not injective, then there is an essential simple loop in $\Sigma$ that represents an element of the kernel of $F_{*}$.

When the map $F$ is an embedding, this follows from the loop theorem of Papakyriakopoulos (see, for instance, Hempel [6]).

The simple loop conjecture is known to hold when the target 3-manifold is a Seifert fibered 3-manifold or a graph 3-manifold, by the work of Hass [4] and Rubinstein and Wang [11], ${ }^{1}$ respectively. An analogous result for maps between surfaces is due to Gabai [3].

The goal of this paper is the following result.

Theorem 1 The simple loop conjecture holds when the target 3-manifold admits a geometric structure modeled on Sol.

\footnotetext{
${ }^{1}$ It is unclear whether the techniques of [11] apply to Sol manifolds, though they seem to be implicitly ruling them out (see, for instance, [11, Lemma 1.0.2]). At any rate, the techniques in this paper offer a substantially different approach to the problem.
} 
If $M$ is a 3 -manifold that is finitely covered by a torus bundle over $S^{1}$, then $M$ admits a geometric structure modeled on one of Euclidean 3-space, Nil, or Sol. Since all compact Euclidean and Nil manifolds are Seifert fibered (see [12]), we obtain the following corollary.

Corollary 2 The simple loop conjecture holds when the target 3-manifold is finitely covered by a torus bundle over $S^{1}$.

This document is organized as follows. In Section 2 we give some definitions and notation for the objects that will be studied. Section 3 contains a brief survey of which compact 3-manifolds admit geometric structures modeled on Sol. This entails a refinement of a classification given by Scott in [12], and reduces the problem at hand to studying maps from closed surfaces into certain kinds of torus bundles over $S^{1}$ and orientable torus semi-bundles. In Sections 4 and 5 we give proofs of the simple loop conjecture for these two types of 3-manifold, respectively. We conclude in Section 6 with some remarks regarding how the results presented here relate to a group-theoretic formulation of the simple loop conjecture, and we show that it fails to hold when the target group is metabelian.

Acknowledgments The author is extremely grateful to Jason Manning for his thoughtful advice, friendly critique, and patience. Additional thanks are due to Alan Reid for pointing out the connection between Example 18 and Casson's construction in [8].

\section{Definitions}

If $M$ is a connected manifold, the orientation character of $M$ is a homomorphism $\rho_{M}: \pi_{1} M \rightarrow \mathbb{Z} / 2$ whose value on $b \in \pi_{1} M$ is nontrivial if and only if some (and hence any) loop in $M$ representing $b$ is orientation reversing. (Equivalently, $\rho_{M}(b)$ is nontrivial if and only if $b$ acts on the universal cover of $M$ by an orientation reversing homeomorphism.) A manifold is orientable if and only if its orientation character is trivial.

If $M$ and $N$ are connected manifolds with orientation characters $\rho_{M}$ and $\rho_{N}$, a map $F: M \rightarrow N$ is called 2-sided if $\rho_{N} \circ F_{*}=\rho_{M}$. Otherwise $F$ is 1 -sided. Hence $F$ is 2 -sided if and only if it takes orientation preserving loops in $M$ to orientation preserving loops in $N$, and likewise for orientation reversing loops. There are other (equivalent) definitions of 2-sidedness for immersions of manifolds, but since most of the arguments in this paper involve the fundamental groups of the manifolds in question, the given definition will be more useful. 
We will call a loop in a manifold $M$ essential if it is neither nullhomotopic nor homotopic into the boundary of $M$. Loops that are not essential will be called inessential.

For a space $X$, we write $|X|$ to denote the number of connected components of $X$. For a compact surface $\Sigma$ with $L \subset \Sigma$ an embedded closed 1-manifold, we will write $\Sigma \backslash L$ to denote the metric completion of $\Sigma \backslash L$ (with respect to some choice of complete metric on $\Sigma$ ). Thus $\Sigma \rrbracket L$ is the space obtained by gluing copies of $S^{1}$ onto the open ends of $\Sigma \backslash L$.

We refer the reader to [12] for an explanation of what it means for a manifold to admit a geometric structure, as well as some basic facts about the Euclidean, Nil, and Sol geometries. In particular, we will need the following two results.

Theorem 3 [12, Theorem 5.2] If $M$ is a closed 3-manifold which admits a geometric structure modeled on one of the eight geometries, then the geometry involved is unique.

Corollary 4 (see [12, Theorem 5.3(ii)]) If $M$ is a closed 3-manifold that admits a Seifert fibering, then $M$ does not admit a geometric structure modeled on Sol.

\subsection{Torus bundles and semi-bundles}

By torus bundle we mean a fiber bundle over $S^{1}$ whose fibers are tori. This can also be viewed as a quotient $T \times I /((p, 0) \sim(\phi(p), 1))$, where $T$ is a torus and $\phi: T \rightarrow T$ is a homeomorphism.

For each $i \in\{1,2\}$, let $N_{i}$ be either a twisted $I$-bundle over a torus or a Klein bottle, so that $\partial N_{i} \cong T$. A torus semi-bundle $M=N_{1} \cup_{\phi} N_{2}$ is obtained by gluing $N_{1}$ and $N_{2}$ by a homeomorphism $\phi: \partial N_{1} \rightarrow \partial N_{2}$. Such a 3 -manifold is orientable if and only if both $N_{1}$ and $N_{2}$ are twisted $I$-bundles over a Klein bottle.

If $M$ is a torus semi-bundle, at times we will refer to the middle torus of $M$, which is the image of $\partial N_{1}$ and $\partial N_{2}$ after gluing. We will also make use of maps $\rho_{i}: \pi_{1} N_{i} \rightarrow \mathbb{Z} / 2$, which are the quotients of $\pi_{1} N_{i}$ by the index two subgroup corresponding to the double covers of $N_{i}$ by the product $T \times I$. (This is sometimes called the monodromy of the $I$-bundle $N_{i}$.) Notice that for $b \in \pi_{1} N_{i}, \rho_{i}(b)$ is trivial if and only if $b$ is represented by a loop that is homotopic into $\partial N_{i}$. Furthermore, when $N_{i}$ is a twisted $I$-bundle over a torus (and is therefore nonorientable), $\rho_{i}$ coincides with the orientation character of $N_{i}$.

If $M$ is a torus semi-bundle, then there is a double cover of $M$ that is the union of the two $T \times I$ double covers of $N_{1}$ and $N_{2}$ along their boundaries (via some 
homeomorphism of the torus). This is a torus bundle over a circle, and is in turn covered by $T \times \mathbb{R}$ with deck group $\mathbb{Z}$. Hence $M$ is covered by $T \times \mathbb{R}$ with deck group the infinite dihedral group $D=\left\langle g_{1}, g_{2} \mid g_{1}^{2}=g_{2}^{2}=1\right\rangle$. The induced action on $\mathbb{R}$ is the usual discrete action of $D$ on $\mathbb{R}$, where $g_{1}$ and $g_{2}$ act by reflections about 0 and 1 , respectively. The projection $T \times \mathbb{R} \rightarrow \mathbb{R}$ therefore induces a projection $M \rightarrow I(2,2)$, where $I(2,2)$ is a 1-dimensional orbifold called the mirrored interval. (See [1] for definitions and notation.) It follows that $M$ can be viewed as an orbifold fiber bundle over $I(2,2)$. The generic fibers of this bundle are 2-sided tori in $M$, and the fibers over the mirrored points are the 1 -sided tori or Klein bottles of $M$.

\section{Classification of compact 3-manifolds modeled on Sol}

In [12], Scott gives the following classification of closed 3-manifolds modeled on Sol. (Note that a homeomorphism $\phi: T \rightarrow T$ of a torus is called hyperbolic if $\phi_{*}$ acts on $H_{1}(T ; \mathbb{Z})$ with $\operatorname{tr}(T)^{2}>4$.)

Theorem 5 [12, Theorem 5.3(i)] Let $M$ be a closed 3-manifold. Then $M$ possesses a geometric structure modeled on Sol if and only if $M$ is finitely covered by a torus bundle over $S^{1}$ with hyperbolic monodromy. In particular, $M$ itself is either a bundle over $S^{1}$ with fiber the torus or Klein bottle or is the union of two twisted I-bundles over the torus or Klein bottle.

We refine this classification as follows.

Theorem 6 Let $M$ be a closed 3-manifold. Then $M$ possesses a geometric structure modeled on Sol if and only if one of the following holds:

(1) $M$ is a torus bundle over $S^{1}$ with hyperbolic monodromy.

(2) $M$ is an orientable torus semi-bundle with gluing map (in canonical coordinates) given by $\left(\begin{array}{cc}r & s \\ t & u\end{array}\right)$, where $r$ stu $\neq 0$.

The notion of canonical coordinates on the middle torus of a torus semi-bundle is explained in the definition that precedes Proposition 1.5 of [14].

Proof It is shown in [14] that an orientable torus semi-bundle admits a Sol structure if and only if its gluing map is of the form stated above. Hence to complete the proof we must show that the other types of 3-manifolds mentioned in Scott's classification do not admit geometric structures modeled on Sol. 
Case 1 ( $M$ is a Klein bottle bundle over $S^{1}$ ) Let

$$
B=\left\langle a, b \mid a b a^{-1} b=1\right\rangle
$$

be the fundamental group of a Klein bottle, and let $A=\left\langle a^{2}, b\right\rangle \approx \mathbb{Z} \oplus \mathbb{Z}$ be the normal subgroup of $B$ corresponding to the double cover of the Klein bottle by a torus. The fundamental group of $M$ has the form

$$
\pi_{1} M=\left\langle B, t \mid t x t^{-1}=\phi(x), \forall x \in B\right\rangle
$$

for some automorphism $\phi$ of $B$ coming from a homeomorphism of the Klein bottle.

We now show that every such automorphism of $B$ preserves the subgroup $A$. We first observe that every element of $B$ can be written uniquely as $a^{i} b^{j}$ for $i, j \in \mathbb{Z}$. Since $\phi$ must preserve the commutator subgroup $[B, B]=\left\langle b^{2}\right\rangle$, we have $\phi\left(b^{2}\right)=b^{ \pm 2}$, and a short computation shows that in fact $\phi(b)=b^{ \pm 1}$. It follows that $\phi(a)=a^{i} b^{j}$, where $i, j \in \mathbb{Z}$ and $i$ is odd, since otherwise $\phi$ has image in the proper subgroup $A$. We have

$$
\phi\left(a^{2}\right)=\left(a^{i} b^{j}\right)\left(a^{i} b^{j}\right)=\left(a^{i} a^{i}\right)\left(b^{-j} b^{j}\right)=a^{2 i},
$$

and similarly $\phi^{-1}\left(a^{2}\right)=a^{2 i^{\prime}}$ for some $i^{\prime} \in \mathbb{Z}$. From $a^{2}=\phi^{-1}\left(\phi\left(a^{2}\right)\right)=a^{2 i \cdot i^{\prime}}$ we find that $i \cdot i^{\prime}=1$, and so $i= \pm 1$. In summary, $\phi(b)=b^{ \pm 1}$ and $\phi\left(a^{2}\right)=a^{ \pm 2}$, so $\phi$ preserves the subgroup $A$.

We therefore conclude that $\pi_{1} M$ contains an index 2 subgroup of the form

$$
H=\left\langle A, t\left|t x t^{-1}=\phi\right|_{A}(x), \forall x \in A\right\rangle .
$$

Let $\widehat{M}$ be the double cover of $M$ corresponding to $H$, which is a torus bundle over $S^{1}$ with monodromy $\left.\phi\right|_{A}$. By the argument in the previous paragraph, there is a choice of basis for $A$ so that

$$
\left.\phi\right|_{A}=\left(\begin{array}{cc} 
\pm 1 & 0 \\
0 & \pm 1
\end{array}\right)
$$

Therefore $\left.\phi\right|_{A}$ corresponds to a periodic homeomorphism of the torus, and so $\widehat{M}$ admits a Euclidean structure by [12, Theorem 5.5]. It follows that $M$ does not admit a Sol structure, for if it did the structure could be lifted to a Sol structure on $\hat{M}$, which would violate Theorem 3 .

Case 2 ( $M$ is a Klein bottle semi-bundle) Then $M$ is double covered by a Klein bottle bundle over $S^{1}$ and therefore has a degree 4 cover that is a torus bundle over $S^{1}$ that admits a Euclidean structure. As in the previous case, $M$ does not admit a Sol structure.

Case 3 ( $M$ is a nonorientable torus semi-bundle) Then $M$ is the union of two twisted $I$-bundles $N_{1}$ and $N_{2}$ over a torus or Klein bottle, at least one of which 
(say $N_{1}$ ) is an $I$-bundle over a torus. We will show that $M$ admits a Seifert fibering, and therefore does not admit a Sol structure by Corollary 4 .

Choose an arbitrary Seifert fibration for $N_{2}$; up to isomorphism there are precisely two of these when $N_{2}$ is an $I$-bundle over a Klein bottle (see [5], for instance) and infinitely many when $N_{2}$ is an $I$-bundle over a torus, as we will show.

If $T$ is a torus, then for any $p / q \in \mathbb{Q} \cup\{\infty\}, T$ can be foliated by $p / q$-curves. This foliation extends to the product Seifert fibration of $T \times I$ by $p / q$-curves in each torus $T \times\{t\}$. Finally, since the covering involution corresponding to the cover $T \times I \rightarrow N_{1}$ preserves the fibration on $T \times I$, it descends to a Seifert fibration of $N_{1}$ so that $\partial N_{1}$ is foliated by $p / q$ curves. Note that this is the one of the "generalized" Seifert fibrations as defined in [12], as the critical fibers are not isolated. In fact, the one-sided torus in $N_{1}$ forms a subsurface of critical fibers.

It follows that a Seifert fibration on $M$ can be constructed by choosing a Seifert fibration on $N_{1}$ so that the foliation of the boundary agrees with the image of the foliation of $\partial N_{2}$ under the gluing map.

\section{Torus bundles}

The first of the two main theorems that will imply Theorem 1 is the following.

Theorem 7 If $M$ is a torus bundle, then the simple loop conjecture holds for $M$.

In fact, a slightly stronger result holds for most surfaces.

Theorem 8 Let $\Sigma$ be a closed surface and let $M$ be a torus bundle. If $\chi(\Sigma)$ is even and negative and $F: \Sigma \rightarrow M$ is a 2-sided map, then there is an essential simple loop in $\Sigma$ that represents an element of ker $F_{*}$. If $\chi(\Sigma)$ is odd then there is no 2-sided map $\Sigma \rightarrow M$.

After we prove Theorem 8, to complete the proof of Theorem 7 it will remain to handle the two cases where $\chi(\Sigma)=0$. The simple loop conjecture is known to hold for maps $\Sigma \rightarrow M$ where $\Sigma$ is a torus and $M$ is any 3 -manifold [4, Section 4.4], and Proposition 11 will deal with the case in which $\Sigma$ is a Klein bottle.

Let $L$ be a (not necessarily connected) 1 -submanifold of a surface $\Sigma$ and let $\alpha$ be an arc in $\Sigma$ with endpoints on $L$ and interior disjoint from $L$. Then surgery of $L$ along $\alpha$ entails fattening $\alpha$ to a strip $I \times I$ with $L \cap(I \times I)=\partial I \times I$, deleting the interior of $\partial I \times I$ from $L$, and gluing in $I \times \partial I$ to $L$. Notice that if $\alpha$ is an arc between two 

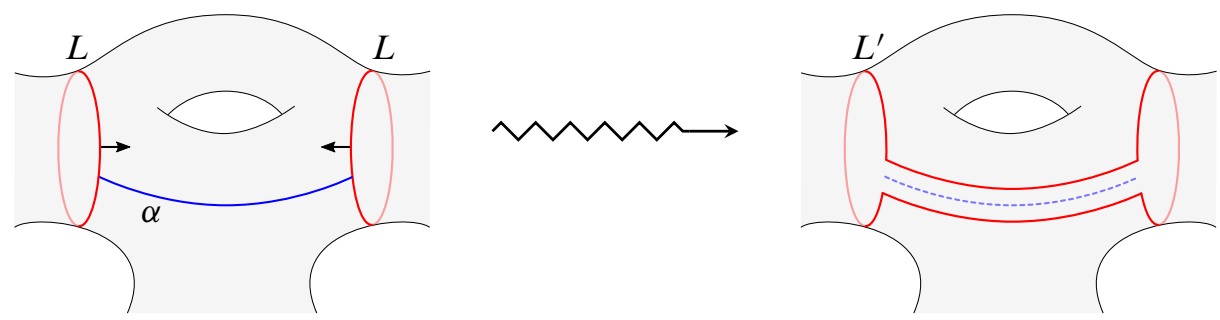

Figure 1: Surgery along $\alpha$ reduces the number of components of $L$ by one

distinct components of $L$, then the result of surgery along $\alpha$ is to connect the two components of $L$ by a bridge, as shown in Figure 1 .

The following can be established by a standard homotopy argument.

Lemma 9 Let $\Sigma$ be a (not necessarily closed) surface, let $J$ denote the open interval $(0,1)$, and let $H: \Sigma \rightarrow J$ be a map that is transverse to a point $r \in J$. If $\alpha$ is an arc that connects two components of $L=H^{-1}(r)$ whose interior is disjoint from $L$, then $H$ can be homotoped in a neighborhood of $\alpha$ so that the preimage of $r$ changes by surgery along $\alpha$.

Lemma 10 Let $\Sigma$ be a closed surface, let $G: \Sigma \rightarrow S^{1}$ be a $\pi_{1}$-surjective map, and choose $q \in S^{1}$. Then $G$ can be homotoped so that the preimage $L=G^{-1}(q)$ is an essential 2-sided simple loop in $\Sigma$.

Proof Choose $G$ within its homotopy class so that $q$ is a regular value of $G$ and $L=G^{-1}(q)$ is a collection of disjoint simple loops in $\Sigma$ with a minimal number of components. Observe that $L$ is 2 -sided but may not be connected. We shall show that the minimality assumption on $L$ along with the assumption that $G$ is $\pi_{1}$-surjective forces $L$ to be connected.

Choose a co-orientation of $q \in S^{1}$ and pull it back to a co-orientation of $L$ in $\Sigma$. We summarize this data by drawing a single arrow orthogonal to each component of $L$ that indicates to which side of each component the co-orientation is pointing, as demonstrated in Figures 1 and 2. When we cut $\Sigma$ along $L$ to obtain $\Sigma \backslash L$, we label the boundary components of the resulting surface with the co-orientations of the components of the $L$ that the boundary components correspond to.

We can homotope $G$ to reduce the number of components of $L$ whenever a component $\Sigma_{0}$ of $\Sigma \rrbracket L$ has two boundary loops that are either both co-oriented into or both co-oriented out of $\Sigma_{0}$. This happens, for instance, whenever $\Sigma_{0}$ has three or more 
boundary components. Start by choosing a simple arc $\alpha \subset \Sigma_{0}$ connecting the two boundary components of $\Sigma_{0}$ with coherent co-orientations, so that $G(\alpha)$ is a nullhomotopic loop in $S^{1}$ based at $q$. If $U$ is a small neighborhood of $\alpha$ in $\Sigma$, then we can homotope $G$ with support in $U$ so that $\left.G\right|_{U}$ is not surjective. Hence $\left.G\right|_{U}$ has image in a subset of $S^{1}$ homeomorphic to $J=(0,1)$, and so we may apply Lemma 9 to $\left.G\right|_{U}$ to obtain a further homotopy of $G$ supported in $U$. This has the effect of surgering $L$ along $\alpha$, which reduces of the number of components of $L$ by one as shown in Figure 1.

Another reduction of $L$ is possible if some component $\Sigma_{0}$ of $\Sigma \rrbracket L$ has only one boundary component. In this case, we homotope $G$ by sending all of $\Sigma_{0}$ past $q$; this homotopy can be taken to be the identity outside of any neighborhood of $\Sigma_{0}$. If $L^{\prime}$ is the preimage of $q$ after the homotopy, then $L^{\prime}$ consists of the same loops as $L$ except for the loop that formed the boundary of $\Sigma_{0}$, which has been eliminated.

It follows that if $G$ is chosen to minimize the number of components of $L$, then every component $\Sigma_{0}$ of $\Sigma \backslash \backslash L$ has exactly two boundary components: one co-oriented into $\Sigma_{0}$ and the other co-oriented out of $\Sigma_{0}$, as shown in Figure 2.

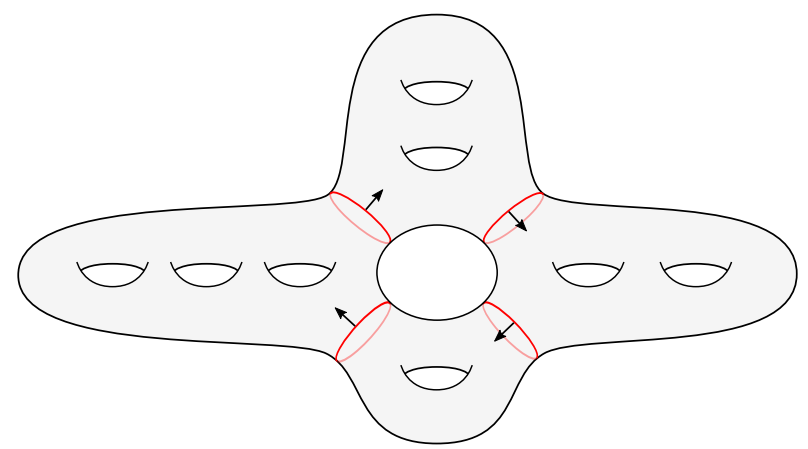

Figure 2: If $L$ has more than one component, then no loop in $\Sigma$ can have a signed intersection of \pm 1 with $L$

We now observe that the homomorphism $G_{*}: \pi_{1} \Sigma \rightarrow \pi_{1} S^{1} \approx \mathbb{Z}$ is given by signed intersection with $L$, where the sign measures whether a loop in $\Sigma$ agrees with the co-orientation of $L$. From the construction of the co-orientation we see that $G_{*}$ must have image $|L| \mathbb{Z} \leq \mathbb{Z}$. Since $G_{*}$ is surjective, we have $|L|=1$, and so $L$ is connected. This completes the proof.

Proof of Theorem 8 Let $P: M \rightarrow S^{1}$ denote the bundle projection of $M$, and let $G=P \circ F: \Sigma \rightarrow S^{1}$.

Case 1 (the map $G$ is $\pi_{1}$-surjective) Applying Lemma 10 to $G$, we may homotope $G$ so that the preimage of a point $q \in S^{1}$ is a 2 -sided simple loop $L \subset \Sigma$ for which 
any loop in $\Sigma \backslash L$ has inessential image under $G$. Since we have that $G(\Sigma \rrbracket L) \subset$ $\left\{S^{1} \backslash q\right\}$, we may use the homotopy lifting property of the fiber bundle $M \rightarrow S^{1}$ to homotope $F$ so that $F(\Sigma \backslash L) \subset M \backslash M_{q}$, where $M_{q}$ is the fiber of $M$ lying above $q$.

Since $M \backslash M_{q}$ is homeomorphic to $T \times I$ and is therefore orientable, it follows from the 2 -sidedness of $F$ that $\Sigma \backslash L$ must be orientable. Therefore $\Sigma \rrbracket L$ is an orientable compact surface with two boundary components, and so $\chi(\Sigma \backslash L)=\chi(\Sigma)$ must be even. This proves the claim that there is no 2 -sided map $\Sigma \rightarrow M$ when $\chi(\Sigma)$ is odd. We may now suppose that $\chi(\Sigma)=2-2 g$, where $g \geq 2$ is an integer. Then $\chi(\Sigma \backslash \backslash L)=$ $2-2 g$, so $\Sigma \backslash L$ is the connect sum of a twice-punctured sphere with $g-1$ tori. It follows that there is an embedded punctured torus $\Sigma_{0}$ in $\Sigma \| L$. The boundary loop $\beta$ of $\Sigma_{0}$ is a separating simple loop in $\Sigma$ whose corresponding element in $\pi_{1} \Sigma$ is the commutator of the elements represented by loops $\gamma$ and $\delta$, as shown in Figure 3 .

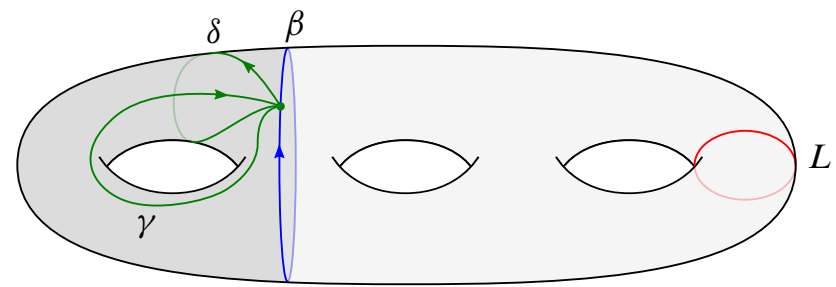

Figure 3: The simple loop $\beta$ in $\operatorname{ker} F_{*}$ is the boundary of the punctured torus $\Sigma_{0} \subset \Sigma$

The loops $\beta, \gamma$ and $\delta$ all have image in $M \backslash M_{q}$, and since $M \backslash M_{q}$ has abelian fundamental group it follows that $F_{*}[\beta]$ is trivial in $\pi_{1} M$. Thus $\beta$ is the desired essential simple loop in the kernel of $F_{*}$. (A similar argument shows that any essential separating loop in $\Sigma \rrbracket L$ must represent an element of ker $F_{*}$.)

Case 2 (the map $G$ is not $\pi_{1}$-surjective) In this case, either $G_{*}$ is the zero map or it has image $n \mathbb{Z} \leq \mathbb{Z} \approx \pi_{1} S^{1}$ for some $n \neq 0, \pm 1$.

If $G_{*}$ is the zero map, then $G$ is homotopic to a constant map, and the homotopy can be lifted to a homotopy of $F$ so that the resulting image of $\Sigma$ is contained in a torus fiber $M_{p}$ of $M$. Since $M_{p}$ is an orientable 2-sided submanifold of $M$, by the 2 -sidedness of $F$ we have that $\Sigma$ is orientable, and so $\chi(\Sigma)$ cannot be odd. If $\chi(\Sigma) \leq-2$ then there is an essential separating loop in $\Sigma$, and we argue as above that such a loop represents an element of ker $F_{*}$.

If instead $G_{*}$ has image a finite-index subgroup $n \mathbb{Z} \leq \mathbb{Z}$, then $p_{*}^{-1}(n \mathbb{Z})$ is a proper finite-index subgroup of $\pi_{1} M$ and $F$ lifts to the corresponding cover $\tilde{M} \rightarrow M$. Since $\tilde{M}$ must also be a torus bundle over a circle and the projection $\widetilde{M} \rightarrow M$ is $\pi_{1}$-injective, we may replace $M$ by $\tilde{M}$ and $F$ by its lift and appeal to Case 1 . 
The following result will complete the proof of Theorem 7 .

Proposition 11 Let $K$ be a Klein bottle and let $G$ be an infinite torsion-free group. If $f: \pi_{1} K \rightarrow G$ is a homomorphism with nontrivial kernel, then there is an essential simple loop in $K$ that represents an element of $\operatorname{ker} f$.

Proof We proceed by reducing to the case in which $f$ has image an infinite cyclic subgroup of $G$. Write the fundamental group of $K$ as

$$
\pi_{1} K=\left\langle a, b \mid a b a^{-1} b=1\right\rangle,
$$

and let $H=\left\langle a^{2}, b\right\rangle \leq \pi_{1} K$ be the index 2 subgroup of $\pi_{1} K$ corresponding to the double cover of $K$ by a torus. The kernel of $\left.f\right|_{H}$ must be nontrivial: for if $x \in \operatorname{ker} f_{*}$ is not the identity then $x^{2} \in H \cap \operatorname{ker} f_{*}$ is also not the identity. Hence $\left.f\right|_{H}$ is a noninjective map from a rank 2 free-abelian group to a torsion-free group, and so the image of $\left.f\right|_{H}$ is either trivial or infinite cyclic. If $f(H)=1$, then since $f(a)^{2}=f\left(a^{2}\right)=1$ and $M$ is torsion-free, $f(a)$ must be trivial. In this case $f$ is the trivial map and we're done. If $f(H)$ is infinite cyclic, then $f\left(\pi_{1} K\right)$ is a virtually infinite cyclic torsion-free group, and so must be infinite cyclic; see, for instance, [13, Theorem 5.12].

Therefore we may replace $f$ by a surjective map $f^{\prime}: \pi_{1} K \rightarrow \mathbb{Z}$. Since $S^{1}$ is a $K(\mathbb{Z}, 1)$, there is a map $F: K \rightarrow S^{1}$ with $F_{*}=f^{\prime}$, and so Lemma 10 can be applied to obtain an essential 2-sided simple loop $L \subset K$ such that every loop in $K \backslash L$ has inessential image in $S^{1}$. Hence we see that $K \backslash L$ is an annulus, the core of which is an essential simple loop in $K$ that represents an element of ker $f^{\prime}$, and hence of ker $f$.

\section{Torus semi-bundles}

The following theorem, together with Theorem 7, will establish Theorem 1.

Theorem 12 If $M$ is an orientable torus semi-bundle that admits a geometric structure modeled on Sol, then the simple loop conjecture holds for $M$.

As in the torus bundle case, we have a slightly stronger statement for maps from surfaces of sufficiently large genus into orientable torus semi-bundles.

Theorem 13 Let $\Sigma$ be a closed surface and let $M$ be an orientable torus semi-bundle. If $\chi(\Sigma)<-2$ and $F: \Sigma \rightarrow M$ is a 2-sided map, then there is an essential simple loop in $\Sigma$ that represents an element of ker $F_{*}$. 
To prove the theorem, we will employ the following two lemmas, which allow us to homotope maps from surfaces to torus semi-bundles into a simplified position.

Lemma 14 Let $M$ be an orientable torus semi-bundle with middle torus $S \subset M$, let $\Sigma$ be a (not necessarily closed) surface, and let $F: \Sigma \rightarrow M$ be a map that is transverse to $S$. Suppose that $\alpha \subset \Sigma$ is a simple arc that connects two distinct components of $L=F^{-1}(S)$ whose interior is disjoint from $L$, and that $F(\alpha)$ is homotopic (rel endpoints) into $S$. Then $F$ can be homotoped in a neighborhood of $\alpha$ so that the preimage of $S$ changes by surgery along $\alpha$.

Proof Let $U$ be a tubular neighborhood of $\alpha$ in $\Sigma$ that does not intersect any components of $L$ except the two that are connected by $\alpha$. Since $F(\alpha)$ is homotopic into $S$, after possibly shrinking $U$ we can homotope $F$ with support in $U$ so that $\left.F\right|_{U}$ has image that does not intersect either of the 1-sided surfaces that are the zero sections of the twisted $I$-bundles that were used to construct $M$.

It follows that $\left.F\right|_{U}$ has image in a subset of $M$ that is homeomorphic to $T \times J$, where $T$ is a torus and $J=(0,1)$. Let $P: T \times J \rightarrow J$ denote the projection onto the second factor, and let $r \in J$ be the image of $S$. Then $\left.P \circ F\right|_{U}: U \rightarrow J$ satisfies the assumptions of Lemma 9, so we may apply it to obtain a homotopy of $\left.P \circ F\right|_{U}$ after which $L$ has been surgered along $\alpha$. Since $T \times J \rightarrow J$ is a fiber bundle, we can lift the homotopy of $\left.P \circ F\right|_{U}$ to a homotopy of $\left.F\right|_{U}$, and from that we obtain a homotopy of $F$ supported in $U$, as desired.

Lemma 15 Let $M$ be an orientable torus semi-bundle with middle torus $S \subset M$, let $\Sigma$ be a closed surface with $\chi(\Sigma)<0$, and let $F: \Sigma \rightarrow M$ be a (2-sided) map that injects on simple loops (that is, there are no elements represented by simple loops in the kernel of $F_{*}$ ). Then $F$ can be homotoped so that $L=F^{-1}(S)$ is either empty or is a collection of parallel 2-sided separating essential simple loops in $\Sigma$.

Figure 4 shows a typical picture of $L \subset \Sigma$ when $L \neq \varnothing$.

Proof In the notation of Section 2.1, let $M=N_{1} \cup_{\phi} N_{2}$ with monodromies

$$
\rho_{i}: \pi_{1} N_{i} \rightarrow \mathbb{Z} / 2 \text {. }
$$

Choose $F$ within its homotopy class so that $F$ is transverse to $S$ and so that $L=$ $F^{-1}(S)$ is a minimal collection of 2-sided simple loops in $\Sigma$.

Step 1 First, suppose that some component $\Sigma_{0}$ of $\Sigma \backslash L$ has three or more boundary components. Let $C_{1}, C_{2}, C_{3}$ be three of the boundary components of $\Sigma_{0}$. (Since $S$ 


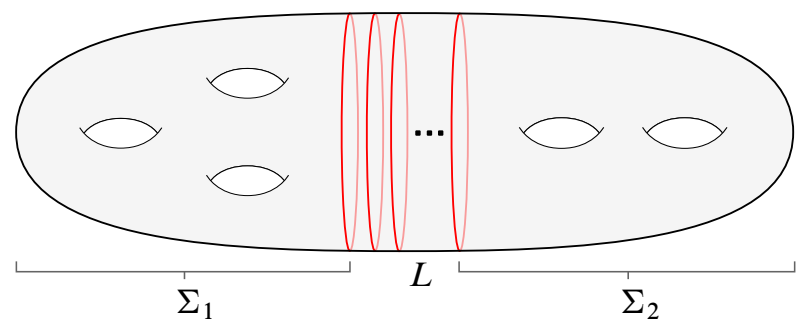

Figure 4: The multicurve $L$ is a collection of parallel loops separating $\Sigma$ into a collection of annuli along with two punctured surfaces, $\Sigma_{1}$ and $\Sigma_{2}$

separates $M$, no two of the $C_{i}$ correspond to the same component of $L$.) Choose a basepoint $q \in S$; after a homotopy of $F$ supported in a tubular neighborhood of the $C_{i}$, we may assume that each $C_{i}$ contains a point $p_{i}$ for which $F\left(p_{i}\right)=q$. In $\Sigma_{0}$ choose simple arcs $\alpha$ from $p_{1}$ to $p_{2}, \alpha^{\prime}$ from $p_{2}$ to $p_{3}$, and $\alpha^{\prime \prime}$ from $p_{1}$ to $p_{3}$ such that $\alpha^{\prime \prime}$ is path-homotopic to the concatenation of $\alpha$ and $\alpha^{\prime}$, as shown in Figure 5.

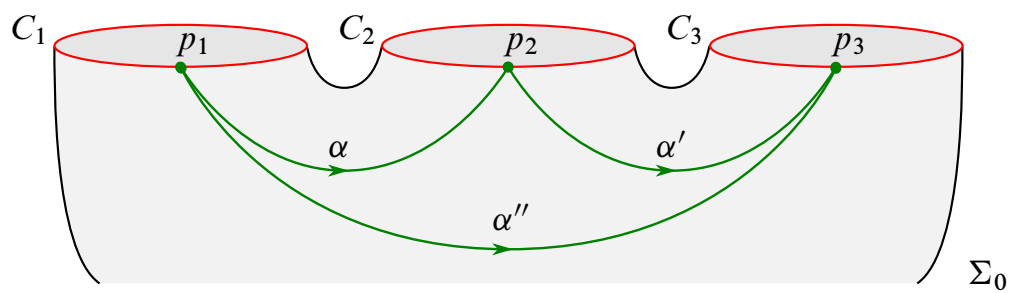

Figure 5: The arcs $\alpha, \alpha^{\prime}$, and $\alpha^{\prime \prime}$ joining the boundary components of $\Sigma_{0}$

By construction, each of $F(\alpha), F\left(\alpha^{\prime}\right)$ and $F\left(\alpha^{\prime \prime}\right)$ are loops in $M$ based at $q$, and without loss of generality all three lie in $N_{1}$. It follows that $\rho_{1}[F(\alpha)], \rho_{1}\left[F\left(\alpha^{\prime}\right)\right]$ and $\rho_{1}\left[F\left(\alpha^{\prime \prime}\right)\right]$ are elements in $\mathbb{Z} / 2$ with $\rho_{1}[F(\alpha)]+\rho_{1}\left[F\left(\alpha^{\prime}\right)\right]=\rho_{1}\left[F\left(\alpha^{\prime \prime}\right)\right]$, and so one of the three elements must be trivial in $\mathbb{Z} / 2$. Hence one of the arcs (say $\alpha$ ) in $\Sigma_{0}$ has image under $F$ that is homotopic into $\partial N_{1}=S$, and so by Lemma 14 we can homotope $F$ so that the result on $L$ is surgery along $\alpha$, which reduces the number of components of $L$.

Step 2 Next, suppose that some component $\Sigma_{0}$ of $\Sigma \backslash L$ has two boundary components and is not an annulus. As in the previous step, we can homotope $F$ in a neighborhood of $\partial \Sigma_{0}$ so that each boundary component has a point $p_{i}(i=1,2)$ that maps to the basepoint $q \in S$. Without loss of generality we assume that $F\left(\Sigma_{0}\right) \subset N_{1}$. There are two cases to consider. 
Case 2A (there is a simple loop $\alpha \subset \Sigma_{0}$ based at $p_{1}$ with $\rho_{1}[F(\alpha)]$ nontrivial in $\mathbb{Z} / 2$ ) Homotope $\alpha$ in $\Sigma_{0}$ so that $\alpha$ becomes the concatenation of two simple arcs $\alpha^{\prime}$ and $\alpha^{\prime \prime}$ from $p_{1}$ to $p_{2}$, as shown in Figure 6 .

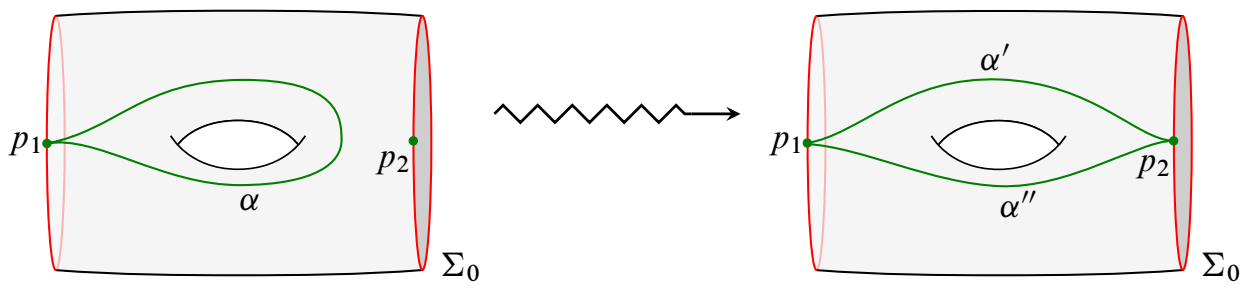

Figure 6: Pulling $\alpha$ towards $p_{2}$ and viewing it as two arcs

It follows that $F\left(\alpha^{\prime}\right)$ and $F\left(\alpha^{\prime \prime}\right)$ are loops in $N_{1}$ based at $q$, and since $\rho_{1}\left[F\left(\alpha^{\prime}\right)\right]+$ $\rho_{1}\left[F\left(\alpha^{\prime \prime}\right)\right]=\rho_{1}[F(\alpha)]$ is nontrivial in $\mathbb{Z} / 2$, one of $\rho_{1}\left[F\left(\alpha^{\prime}\right)\right]$ and $\rho_{1}\left[F\left(\alpha^{\prime \prime}\right)\right]$ must be trivial. As before, an arc with trivial image can be used (Lemma 14) to homotope $F$ and surger $L$, which reduces the number of components of $L$ by one.

Case 2B (for every simple loop $\alpha \subset \Sigma_{0}$ based at $p_{1}, \rho_{1}[F(\alpha)]$ is trivial) Since we assumed $\Sigma_{0}$ is not an annulus, it is a twice-punctured orientable surface of genus greater than 0 . It follows that we can find two simple loops $\gamma$ and $\delta$ in $\Sigma_{0}$ whose commutator in $\pi_{1} \Sigma_{0}$ is represented by a simple loop $\beta$; see Figure 7 .

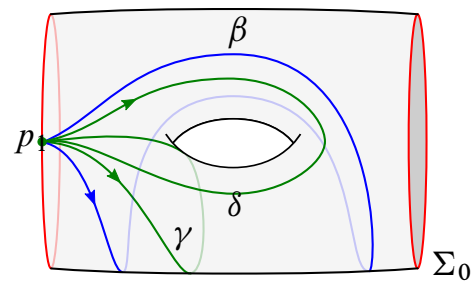

Figure 7: The simple loop $\beta$ represents the commutator of $[\gamma]$ and $[\delta]$

Since $[\beta],[\gamma],[\delta] \in \pi_{1} \Sigma_{0}$, all have trivial image under $\rho_{1} \circ F_{*}, \rho_{1}[F(\beta)], \rho_{1}[F(\gamma)]$, and $\rho_{1}[F(\delta)]$ must lie in the subgroup of $\pi_{1} N_{1}$ corresponding to the boundary $S$. But since $\pi_{1} S$ is abelian, the commutator $F_{*}[\beta]$ is trivial. This contradicts the assumption that $F$ injects on simple loops, and so it is impossible that $\rho_{1} \circ F_{*}$ is trivial on every simple loop in $\Sigma_{0}$.

We conclude that the number of components of $L$ can be reduced whenever some component of $\Sigma \rrbracket L$ has exactly two boundary components and is not an annulus. 
Step 3 It follows from the previous two steps that if $F$ is chosen in its homotopy class so that $L$ has a minimal number of components, then $L$ is either empty or every component of $\Sigma \backslash L$ is either an annulus or a surface with exactly one boundary component. The assumption that $\chi(\Sigma)<0$ rules out the possibility that every component of $\Sigma \backslash \backslash L$ is an annulus, and so $\Sigma$ consists of two punctured orientable surfaces connected by some number of annuli.

Proof of Theorem 13 Let $\Sigma$ be a closed surface with $\chi(\Sigma)<-2$, let $M=N_{1} \cup_{\phi} N_{2}$ be a torus semi-bundle, and let $F: \Sigma \rightarrow M$ be a 2 -sided map. By Lemma 15, we may assume that $F$ has been homotoped so that $L=F^{-1}(S)$ is either empty or is a collection of parallel curves as in Figure 4. (According to the lemma, if this is not possible then we can already find a simple loop in $\operatorname{ker} F_{*}$.)

If $L=\varnothing$ then without loss of generality $F$ has image in $N_{1}$, which is homotopy equivalent to a Klein bottle. Since $\pi_{1} N_{1}$ does not contain the fundamental group of any surface of negative Euler characteristic, the induced map $\pi_{1} \Sigma \rightarrow \pi_{1} N_{1}$ has nontrivial kernel. Using Gabai's result [3], we conclude that there is a simple loop in the kernel of $F_{*}$.

We now consider the case in which $L \neq \varnothing$. If $\Sigma_{1}$ and $\Sigma_{2}$ are the two non-annular subsurfaces of $\Sigma$ as shown in Figure 4, then

$$
\chi\left(\Sigma_{1}\right)+\chi\left(\Sigma_{2}\right)=\chi(\Sigma) .
$$

It follows that either $\chi\left(\Sigma_{1}\right)<-1$ or $\chi\left(\Sigma_{2}\right)<-1$.

Without loss of generality, we will henceforth assume that $\chi\left(\Sigma_{1}\right)<-1$ and that $F\left(\Sigma_{1}\right) \subset N_{1}$.

If $f=\rho_{1} \circ\left(\left.F\right|_{\Sigma_{1}}\right)_{*}: \pi_{1}\left(\Sigma_{1}\right) \rightarrow \mathbb{Z} / 2$, then since $F$ sends $\partial \Sigma_{1}$ (which is a component of $L$ ) into $S$, we have $f\left[\partial \Sigma_{1}\right]=0$. It follows that $f$ represents a class in $H^{1}\left(\Sigma_{1}, \partial \Sigma_{1} ; \mathbb{Z} / 2\right)$. If $f$ represents the trivial class, then all of $F\left(\Sigma_{1}\right)$ is homotopic into $S$, and we can homotope $F$ to send all of $\Sigma_{1}$ past $S$ and reduce the number of components of $L$, contradicting the assumption that $F$ has already been homotoped to minimize the number of components. Therefore $f$ is nontrivial in $H^{1}\left(\Sigma_{1}, \partial \Sigma_{1} ; \mathbb{Z} / 2\right)$, and so by Lefschetz duality, there is a nontrivial homology class $f_{*} \in H_{1}\left(\Sigma_{1} ; \mathbb{Z} / 2\right)$ for which the value of $f$ on any loop $\alpha$ based on $\partial \Sigma_{1}$ is given by the signed intersection $(\bmod 2)$ of $\alpha$ with any 1 -chain representing $f_{*}$.

Let $\ell$ be a simple loop in $\Sigma_{1}$ that represents $f_{*}$. (A simple loop representative exists by [10].) Since $f_{*}$ is nontrivial, $\ell$ is essential and every loop in $\Sigma_{1} \backslash \ell$ is in the kernel of $f$ and therefore has image in $N_{1}$ that is homotopic into $S$. The fact that $\chi\left(\Sigma_{1}\right)<-1$ implies that $\Sigma_{1} \| \ell$ is homeomorphic to a closed surface of genus at least one with 
three open discs removed. As in the proof of Theorem 8, we can find an embedded punctured torus $P$ in $\Sigma_{1} \backslash \ell$ whose boundary $\beta$ represents the commutator of simple loops $\gamma$ and $\delta$ contained in $P$. Since $[\beta],[\gamma]$ and $[\delta]$ all have image under $F_{*}$ in the abelian subgroup $\pi_{1} S \leq \pi_{1} M$, we conclude that $\beta$ is the desired simple loop representing an element of ker $F_{*}$.

With Proposition 11 and the proof of the simple loop conjecture when the domain is a torus given in [4], we will complete the proof of Theorem 12 with the following special case.

Lemma 16 Let $\Sigma$ denote the closed orientable surface with $\chi(\Sigma)=-2$. If $M$ is an orientable torus semi-bundle and $F: \Sigma \rightarrow M$ is a (2-sided) map, then either there is an essential simple loop in $\operatorname{ker} F_{*}$ or $M$ does not admit a geometric structure modeled on Sol.

Proof By Lemma 15, we can homotope $F$ so that the preimage $L=F^{-1}(S)$ of the middle torus of $M$ is a minimal collection of parallel curves in $\Sigma$ as in Figure 4. As in the proof of Theorem 13 we may also assume that $L \neq \varnothing$, so $L$ separates $\Sigma$ into punctured tori $\Sigma_{1}$ and $\Sigma_{2}$ along with a collection of $n=|L|-1$ annuli.

Case $n=0$ In this case, $L$ is connected and separates $\Sigma$ into punctured tori $\Sigma_{1}$ and $\Sigma_{2}$. We can write the fundamental group of $\Sigma$ as

$$
\pi_{1} \Sigma=\left\langle a_{1}, b_{1}, a_{2}, b_{2} \mid\left[a_{1}, b_{1}\right]=\left[a_{2}, b_{2}\right]\right\rangle,
$$

where $a_{i}$ and $b_{i}$ are the generators of the fundamental group of $\Sigma_{i}$. The fundamental group of $M$ has presentation

$$
\pi_{1} M=\left\langle x_{1}, y_{1}, x_{2}, y_{2} \mid x_{i} y_{i} x_{i}^{-1} y_{i}=1, x_{1}^{2}=x_{2}^{2 r} y_{2}^{t}, y_{1}=x_{2}^{2 s} y_{2}^{u}\right\rangle,
$$

where $x_{i}$ and $y_{i}$ are the generators of the fundamental group of the twisted $I$-bundle over a Klein bottle $N_{i}$, and $M$ has been constructed by gluing $N_{1}$ to $N_{2}$ via a homeomorphism $\partial N_{1} \rightarrow \partial N_{2}$ whose matrix is

$$
\left(\begin{array}{ll}
r & s \\
t & u
\end{array}\right) \in \mathrm{GL}_{2}(\mathbb{Z})
$$

with respect to the bases $\left\langle x_{i}^{2}, y_{i}\right\rangle$ of the fundamental groups of the boundaries of the $N_{i}$. By the definition of $L$ we see that $F$ restricts to a proper map of $\Sigma_{i}$ into $N_{i}$, and so $F_{*}\left(a_{i}\right)$ and $F_{*}\left(b_{i}\right)$ must lie in $\left\langle x_{i}, y_{i}\right\rangle$ for $i=1,2$. The subgroup $\left\langle x_{i}, y_{i}\right\rangle$ of $\pi_{1} M$ is isomorphic to the fundamental group of a Klein bottle, and its commutator subgroup 
is infinite cyclic with generator $y_{i}^{2}$. Hence the commutators $\left[a_{i}, b_{i}\right]$ are mapped to even powers of $y_{i}$, and from the relation in $\pi_{1} \Sigma$ we obtain an equation

$$
y_{1}^{2 k_{1}}=y_{2}^{2 k_{2}}
$$

for some integers $k_{1}$ and $k_{2}$. Applying the rightmost relation of the presentation of $\pi_{1} M$ given above, we have

$$
x_{2}^{4 s k_{1}} y_{2}^{2 u k_{1}}=y_{2}^{2 k_{2}} .
$$

Since this is an equation in $\left\langle x_{2}^{2}, y_{2}\right\rangle \approx \mathbb{Z} \oplus \mathbb{Z}$, we can conclude that $4 s k_{1}=0$, and so either $k_{1}=0$ or $s=0$. If $k_{1}=0$, it follows that the curve $L$ (which represents the elements $\left[a_{1}, b_{1}\right]$ and $\left[a_{2}, b_{2}\right]$ in $\pi_{1} \Sigma$ ) has image $y_{1}^{2 k_{1}}=1$, so $L$ is an essential simple loop in the kernel of $F_{*}$. If $s=0$, then by Theorem 6 it follows that $M$ does not admit a geometric structure modeled on Sol.

Case $\boldsymbol{n}>\boldsymbol{0}$ In this case, $L$ has multiple components; we will show that $F$ can be lifted to a torus semi-bundle cover of $M$ in which the preimage of the middle torus is connected, thereby reducing to the case in which $n=0$. Choose points $p_{0}, \ldots, p_{n}$ on the $n+1$ components of $L$, and let $\alpha \subset \Sigma$ be a simple arc with end points at $p_{0}$ and $p_{n}$ whose intersection with $L$ is the points $p_{i}$. For $i=0, \ldots, n-1$ let $\alpha_{i}$ denote the segment of $\alpha$ between $p_{i}$ and $p_{i+1}$, as shown in Figure 8 .

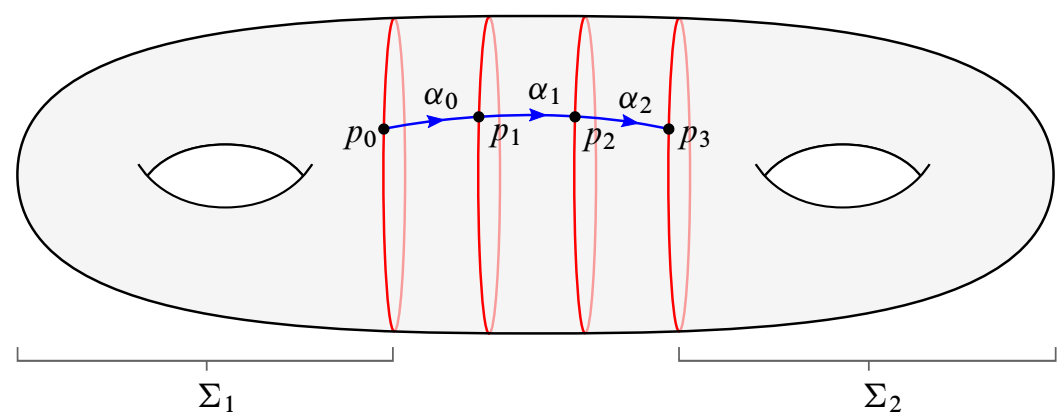

Figure 8: The arc $\alpha$ connecting the points $p_{i}$ in the case $n=3$

By adjusting $F$ by a homotopy that preserves $L$, we may assume that $F\left(p_{i}\right)=q$ for some basepoint $q \in S \subset M$, and so $F\left(\alpha_{i}\right)$ is a loop in $M$ based at $q$ representing an element $w_{i} \in \pi_{1} M$.

In the notation of the previous case, we assume that $F_{*}\left(a_{1}\right)$ and $F_{*}\left(b_{1}\right)$ lie in the subgroup $\left\langle x_{1}, y_{1}\right\rangle \leq \pi_{1} M$, and by the definition of $L$ we have that $w_{i} \in\left\langle x_{j_{i}}, y_{j_{i}}\right\rangle$, where $j_{i}=1$ if $i$ is odd and $j_{i}=2$ if $i$ is even. We may also assume that $w_{i} \notin\left\langle x_{j_{i}}^{2}, y_{j_{i}}\right\rangle$, for if $w_{i} \in\left\langle x_{j_{i}}^{2}, y_{j_{i}}\right\rangle$ then $\alpha_{i}$ is a proper simple arc in a component $\Sigma \| L$ with 
image homotopic into $S$, and we can reduce the number of components of $L$, which contradicts the minimality assumption. If $w=w_{0} \cdots w_{n-1}$, then we have

$$
F_{*}\left(\pi_{1} \Sigma\right) \leq\left\langle x_{1}, y_{1}, w x_{k} w^{-1}, w y_{k} w^{-1}\right\rangle,
$$

where $k=1$ if $n$ is odd and $k=2$ if $n$ is even.

If $D=\left\langle g_{1}, g_{2} \mid g_{1}^{2}=g_{2}^{2}=1\right\rangle$ denotes the infinite dihedral group, then there is a homomorphism $f: \pi_{1} M \rightarrow D$ given by $x_{i} \mapsto g_{i}$ and $y_{i} \mapsto 1$ for $i=1,2$. The cover of $M$ corresponding to $\operatorname{ker} f$ is $T \times \mathbb{R}$ with deck group $D$, as described in Section 2.1. For each $i=0, \ldots, n-1$, since $w_{i} \notin\left\langle x_{j_{i}}^{2}, y_{j_{i}}\right\rangle$ we have $f\left(w_{i}\right)=g_{j_{i}}$, and it follows that $f(w)$ is a reduced word in $D$ of length $n$ starting with $g_{2}$. The image of $\pi_{1} \Sigma$ under the composition $f \circ F_{*}$ is the subgroup

$$
H=\left\langle g_{1}, f(w) g_{k} f(w)^{-1}\right\rangle \leq D,
$$

which itself is isomorphic to the infinite dihedral group. Let $\widehat{M}$ be the quotient of $S \times \mathbb{R}$ by $H$, which is another torus semi-bundle that is the cover of $M$ corresponding to the subgroup $f^{-1}(H)$. Then $\widehat{M}$ contains $n+1$ tori $S_{0}, \ldots, S_{n}$ that are lifts of $S$, and the result of splitting $\widehat{M}$ along these tori is $n$ products $T \times I$ (each of which double-covers $N_{1}$ or $N_{2}$ ) along with two twisted $I$-bundles over a Klein bottle (each of which projects to $N_{1}$ or $N_{2}$ by a homeomorphism). The $S_{i}$ are parallel and one can show that $\widehat{F}^{-1}\left(S_{i}\right)$ is connected for $i=0, \ldots, n$, where $\widehat{F}: \Sigma \rightarrow \hat{M}$ is the lift of $F$ to $\hat{M}$. Hence we can take any of the $S_{i}$ to be the "middle torus" of $\widehat{M}$.

Therefore we may apply the argument of the first case of this proof to $\widehat{F}$ to find either an essential simple loop in ker $\widehat{F}_{*}$ or that $\widehat{M}$ is Seifert fibered. In the former case, an essential simple loop in ker $\widehat{F}_{*}$ is also an essential simple loop in ker $F_{*}$. In the latter, if $\widehat{M}$ is Seifert fibered then it carries a Euclidean or Nil structure, and therefore so does $M$. It follows that $M$ is Seifert fibered as well.

\section{The simple loop conjecture for metabelian groups}

An orientation character on a group $G$ is a homomorphism $\rho_{G}: G \rightarrow \mathbb{Z} / 2$, and an oriented group is a pair $\left(G, \rho_{G}\right)$ where $\rho_{G}$ is an orientation on $G$. When $G$ is the fundamental group of a manifold $M$, we take $\rho_{G}$ to be the orientation character $\rho_{M}$ defined in Section 2. Similarly, one can say what it means for a homomorphism between two oriented groups to be 2 -sided. It then seems natural to ask if the following generalization of the simple loop conjecture holds for a fixed oriented group $G$.

Statement Let $\Sigma$ be a closed surface and let $\left(G, \rho_{G}\right)$ be an oriented group. If $f: \pi_{1} \Sigma \rightarrow G$ is a 2 -sided homomorphism that is not injective, then there is an essential simple loop in $\Sigma$ that represents an element of the kernel of $f$. 
When $G$ is the fundamental group of an aspherical 3-manifold this is equivalent to the simple loop conjecture for 3-manifolds. This statement is known to be false when $G=\operatorname{PSL}(2, \mathbb{C})$ by work of Cooper and Manning [2] and when $G=\operatorname{PSL}(2, \mathbb{R})$ by work of Mann [9]. (In both cases, $G$ carries the trivial orientation character as it is identified with the groups of orientation-preserving isometries of hyperbolic 3-and 2-space, respectively.)

A group is called metabelian if it fits into a short exact sequence of the form

$$
1 \rightarrow A \rightarrow G \rightarrow B \rightarrow 1
$$

where $A$ and $B$ are abelian groups. For example, the fundamental groups of the torus bundles treated in Section 4 are metabelian with $A=\mathbb{Z} \oplus \mathbb{Z}$ and $B=\mathbb{Z}$. One might be led to ask if the group-theoretic version of the simple loop conjecture holds for metabelian groups, and if a technique similar to that of Section 4 can be used to prove it. We provide the following result in this direction.

Theorem 17 Let $\left(G, \rho_{G}\right)$ be an oriented group that fits into an exact sequence of the form

$$
1 \rightarrow A \rightarrow G \rightarrow \mathbb{Z} \rightarrow 1
$$

where $A$ is abelian, and suppose that $A \leq \operatorname{ker} \rho_{G}$. If $\Sigma$ is a closed surface of genus at least two, then the group-theoretic version of the simple loop conjecture holds for $\Sigma$ and $G$.

Proof This is a group-theoretic analogue to the proof of Theorem 8. Let $p: G \rightarrow \mathbb{Z}$ denote the projection map in the short exact sequence. For a surface $\Sigma$ and a $2-$ sided homomorphism $f: \pi_{1} \Sigma \rightarrow G$, we may assume that $f$ is surjective. For if not, then either $f\left(\pi_{1} \Sigma\right)$ lies in $A$ and any separating simple loop in $\Sigma$ represents an element of ker $f$, or $p \circ f$ has nontrivial image and we replace $G$ by $f\left(\pi_{1} \Sigma\right), \rho_{G}$ by $\left.\left(\rho_{G}\right)\right|_{f\left(\pi_{1} \Sigma\right)}, A$ by $A \cap f\left(\pi_{1} \Sigma\right)$, and $\mathbb{Z}$ by $(p \circ f)\left(\pi_{1} \Sigma\right) \approx \mathbb{Z}$.

There is a map $\Sigma \rightarrow S^{1}$ whose induced homomorphism on fundamental groups is $p \circ f$, and by applying Lemma 10 to this map we find a simple nonseparating loop $L \subset \Sigma$ such that every element of $\pi_{1}(\Sigma \backslash L) \leq \pi_{1} \Sigma$ is contained in $\operatorname{ker}(p \circ f)$. By exactness, $f\left(\pi_{1}(\Sigma \rrbracket L)\right)$ is contained in $A$, and the assumptions that $f$ is 2 -sided and that $A \leq \operatorname{ker} \rho_{G}$ imply that $\Sigma \rrbracket L$ must be orientable.

As shown in the proof of Theorem 8 there are essential simple loops $\beta, \gamma$, and $\delta$ in $\Sigma$ representing elements of $\operatorname{ker}(p \circ f)$ and with $[\beta]$ equal to the commutator of $[\gamma]$ and $[\delta]$. By exactness, $f[\beta], f[\gamma]$ and $f[\delta]$ are contained in $A$, and since $A$ is abelian we have that $f[\gamma]$ is trivial. 
We conclude by showing that, despite the previous result, the group-theoretic simple loop conjecture does not hold for all torsion-free metabelian groups. This is a torsionfree version of a finite example due to Casson [8, Section 2].

Example 18 Let $\Sigma$ be a surface of genus $g \geq 2$. We will give a topological construction of the quotient of $\pi_{1} \Sigma$ by its second derived subgroup, which is sometimes called the metabelianization of $\pi_{1} \Sigma$. From the construction we will see that the kernel of $\pi_{1} \Sigma \rightarrow G$ does not contain any elements represented by simple loops in $\Sigma$.

First, let $B=H_{1}(\Sigma)$ (with $\mathbb{Z}$ coefficients understood), let $f_{1}: \pi_{1} \Sigma \rightarrow B$ be the abelianization map, and let $K_{1}=\operatorname{ker} f_{1}$. Let $P: \widehat{\Sigma} \rightarrow \Sigma$ be the cover of $\Sigma$ corresponding to $K_{1}$. Next, let $f_{2}: \pi_{1} \widehat{\Sigma} \rightarrow H_{1}(\hat{\Sigma})$ be the analogous natural map for $\hat{\Sigma}$, and let $K_{2}=\operatorname{ker} f_{2}$. We have $K_{2} \leq \pi_{1} \hat{\Sigma} \approx K_{1} \leq \pi_{1} \Sigma$, and so we identify $K_{2}$ with its image under $P_{*}$ and consider it a subgroup of $\pi_{1} \Sigma$.

Observe that $K_{1}$ does not contain any element of $\pi_{1} \Sigma$ represented by a nonseparating simple loop in $\Sigma$, but does contain every element represented by a separating simple loop in $\Sigma$. Hence every separating simple loop in $\Sigma$ lifts to $\widehat{\Sigma}$; we now show that every such loop lifts to a nonseparating simple loop in $\widehat{\Sigma}$.

We first observe that $B \approx \mathbb{Z}^{2 g}$ is a one-ended group. Since $B$ acts properly on $\hat{\Sigma}$ with compact quotient $\Sigma$, it follows that $\hat{\Sigma}$ is a one-ended space. Any inessential separating simple loop in $\hat{\Sigma}$ must therefore separate $\widehat{\Sigma}$ into a compact piece and a noncompact piece. Hence if $\beta$ is a simple separating loop in $\Sigma$ for which some (and hence any) lift $\hat{\beta}$ of $\beta$ separates $\widehat{\Sigma}$, then $\hat{\beta}$ cuts off a compact subsurface $\widehat{\Sigma}_{\widehat{\beta}} \subset \widehat{\Sigma}$. If $\hat{\beta}^{\prime}$ is another lift of $\beta$, then $\hat{\beta}$ and $\hat{\beta}^{\prime}$ are disjoint, and the regularity of the cover $\hat{\Sigma} \rightarrow \Sigma$ implies that there is a deck transformation of $\hat{\Sigma}$ that takes $\hat{\beta}^{\prime}$ to $\hat{\beta}$. This deck transformation must take $\widehat{\Sigma}_{\hat{\beta}^{\prime}}$ homeomorphically onto $\widehat{\Sigma}_{\widehat{\beta}}$. If one of these subsurfaces is contained in the other (say we have $\widehat{\Sigma}_{\widehat{\beta}^{\prime}} \subset \widehat{\Sigma}_{\widehat{\beta}}$ ), then $\hat{\beta}$ and $\hat{\beta}^{\prime}$ must be parallel. However, this is impossible: for by choosing hyperbolic metrics on $\Sigma$ and $\widehat{\Sigma}$ so that the covering action is by isometries, and choosing $\beta, \hat{\beta}$ and $\hat{\beta}^{\prime}$ to be the unique geodesics in their homotopy classes, we see that if $\hat{\beta}$ and $\hat{\beta}^{\prime}$ are parallel then they are not distinct lifts of $\beta$.

It follows that the subsurfaces $\widehat{\Sigma}_{\widehat{\beta}}$ (as $\widehat{\beta}$ ranges over the lifts of $\beta$ ) must be disjoint. In particular, each such subsurface does not contain any lifts of $\beta$ in its interior. Thus the covering map $\hat{\Sigma} \rightarrow \Sigma$ restricts to a cover of a component of $\Sigma \backslash \beta$ by $\hat{\Sigma}_{\widehat{\beta}}$, and since $\hat{\beta}$ projects to $\beta$ via a homeomorphism, the restricted cover is a homeomorphism. However, this is impossible, as $\hat{\Sigma}_{\widehat{\beta}}$ is not a disk and so must contain a nonseparating simple loop, and this nonseparating loop is a lift of its image under the covering projection. We have already observed that such loops do not lift from $\Sigma$ to $\widehat{\Sigma}$, and so 
from this contradiction we conclude that $\hat{\beta}$ (and hence every lift of $\beta$ to $\hat{\Sigma}$ ) must be nonseparating.

It follows that $K_{2}$ does not contain any elements represented by simple loops of $\Sigma$, since the nonseparating simple loops in $\Sigma$ are homologically nontrivial, and the separating simple loops of $\Sigma$ lift to homologically nontrivial loops in $\widehat{\Sigma}$. Hence if we let $G=\pi_{1} \Sigma / K_{2}$ and let $f: \pi_{1} \Sigma \rightarrow G$ be the quotient map, then $f$ is a noninjective map with no elements represented by essential simple loops in its kernel. If $A=\pi_{1} \widehat{\Sigma} / K_{2} \approx H_{1}(\widehat{\Sigma})$, then $A$ is abelian and we have

$$
G / A=\left(\pi_{1} \Sigma / K_{2}\right) /\left(\pi_{1} \hat{\Sigma} / K_{2}\right) \approx \pi_{1} \Sigma / \pi_{1} \hat{\Sigma} \approx \pi_{1} \Sigma / K_{1} \approx H_{1}(\Sigma),
$$

which is also abelian. Thus we see that $G$ is metabelian, for it fits into the short exact sequence

$$
1 \rightarrow H_{1}(\widehat{\Sigma}) \rightarrow G \rightarrow H_{1}(\Sigma) \rightarrow 1,
$$

and so we have constructed the desired group $G$ and map $f: \pi_{1} \Sigma \rightarrow G$.

\section{References}

[1] D Cooper, CD Hodgson, S P Kerckhoff, Three-dimensional orbifolds and conemanifolds, MSJ Memoirs 5, Math. Soc. Japan, Tokyo (2000) MR1778789

[2] D Cooper, J F Manning, Non-faithful representations of surface groups into SL(2, C) which kill no simple closed curve, Geom. Dedicata 177 (2015) 165-187 MR3370029

[3] D Gabai, The simple loop conjecture, J. Differential Geom. 21 (1985) 143-149 MR806708

[4] J Hass, Minimal surfaces in manifolds with $S^{1}$ actions and the simple loop conjecture for Seifert fibered spaces, Proc. Amer. Math. Soc. 99 (1987) 383-388 MR870806

[5] A Hatcher, Notes on basic 3-manifold topology, course notes, Cornell University (2007) Available at https://www.math.cornell.edu/ hatcher/3M/ 3Mdownloads .html

[6] J Hempel, 3-Manifolds, Ann. of Math. Studies 86, Princeton Univ. Press (1976) MR0415619

[7] R Kirby, Problems in low-dimensional topology, from "Geometric topology" (W H Kazez, editor), AMS/IP Stud. Adv. Math. 2, Amer. Math. Soc., Providence, RI (1997) 35-473 MR1470751

[8] C Livingston, Maps of surface groups to finite groups with no simple loops in the kernel, J. Knot Theory Ramifications 9 (2000) 1029-1036 MR1807606

[9] K Mann, A counterexample to the simple loop conjecture for $\operatorname{PSL}(2, \mathbb{R})$, Pacific J. Math. 269 (2014) 425-432 MR3238484 
[10] MD Meyerson, Representing homology classes of closed orientable surfaces, Proc. Amer. Math. Soc. 61 (1976) 181-182 MR0425967

[11] J H Rubinstein, $\mathbf{S}$ Wang, $\pi_{1}$-injective surfaces in graph manifolds, Comment. Math. Helv. 73 (1998) 499-515 MR1639876

[12] P Scott, The geometries of 3-manifolds, Bull. London Math. Soc. 15 (1983) 401-487 MR705527

[13] P Scott, T Wall, Topological methods in group theory, from "Homological group theory" (C T C Wall, editor), London Math. Soc. Lecture Note Ser. 36, Cambridge Univ. Press (1979) 137-203 MR564422

[14] H Sun, S Wang, J Wu, Self-mapping degrees of torus bundles and torus semi-bundles, Osaka J. Math. 47 (2010) 131-155 MR2666128

Department of Mathematics, Cornell University

310 Malott Hall, Ithaca, NY 14853, United States

zemke@math. cornell. edu

http://www . math. cornell. edu/ zemke/

Received: 30 December 2015 Revised: 14 March 2016 
